\title{
Research on the Multi-scale Fuzzy Entropy based on Index Energy
}

\author{
Zhonglin Wei ${ }^{1, a}$, Liyuan $\mathrm{Ma}^{2, \mathrm{~b}}$, Xinhan Cui ${ }^{3, \mathrm{c}}$ \\ ${ }^{1}$ Mechanical Engineering College, Shijiazhuang, 050003, China \\ ${ }^{2}$ Mechanical Engineering College, Shijiazhuang, 050003, China \\ ${ }^{3}$ Mechanical Engineering College, Shijiazhuang, 050003, China \\ aemail:wzl_123@126.com, bemail:maliyuan1962@163.com, 'email:cui_2k@163.com
}

Keywords: Multi-scale Fuzzy Entropy; Index Entropy; Empirical Mode Decomposition

\begin{abstract}
In order to improve the accuracy of feature extraction, a Multi-scale Fuzzy Entropy algorithm is proposed based on Entropy theory. The new algorithm combines the concept of Fuzzy Entropy and Multi-scale Entropy. Aimed at the shortage of Empirical mode decomposition (EMD), Index Energy is selected as evaluation index, and a new feature extraction method is designed to dispose vibration signal. Experiment shows that the new method can effectively distinguish different condition of rotor vibration signal and can significantly improve the effect of feature extraction.
\end{abstract}

\section{Introduction}

As the core technology of fault diagnosis, the quality of feature extraction directly influences the effect of pattern recognition. Hence, choosing a suitable method to extract feature information which can effectively reflect the signal's innate-character becomes the key point of feature extraction's field. Traditional time-frequency analysis method cannot obtain the feature information which contained in signals. Shannon putted forward the concept of Information Entropy in 1948 to describe the measurement of system's disorder in microscopic particle and introduced to the field of information theory. With the development of entropy theory and wide application, Pincus put forward Approximate Entropy to analyze complexity in time series' [1]. And Richman proposed the concept of Sample Entropy in 2000 [2]. As an improved algorithm, since the proposing of Sample Entropy, it immediately attracted extensive attention. Based on the concept of Sample Entropy, Scholar gave the concept of Fuzzy Entropy and Multi-scale Entropy. Fuzzy Entropy utilizes the pattern of exponential function's fuzzily to dispose entropy's mutation in the process of counting for using unit step function [3]. Multi-scale Entropy starts with different scales analysis, meanwhile avoiding the sole scale reflect serial information one-sided, and can dig the order's deep information.

\section{Formation of Multi-scale Fuzzy Entropy}

For effectively distinguish the diversity in signal, we need to make the inherent difference maximize. After giving an overall analysis to the general entropy theories, we select the Fuzzy Entropy as basic technique for signal's feature entropy extraction, and use Multi-scale analysis as reference to introduce Multi-scale Entropy to construct the Multi-scale Fuzzy Entropy. Utilizing Multi-scale Fuzzy Entropy to dispose the vibration signal in different condition and obtain the feature information, then compare the extraction effects in different scale to search the best analysis scale to furthest distinguish the weak change of complexity in different condition.

Multi-scale Fuzzy Entropy concurrently has the character of Fuzzy Entropy and Multi-scale Entropy. So the counting process vastly fuses the operation characteristic of both. The calculated flow is as follow:

(1) Disposing the signal with coarse graining, and combining with resemble tolerance $r$ and embed mode dimension $n$ to construct coarse vector. 


$$
x_{j}(\lambda)=\frac{1}{\lambda} \sum_{i=(j-1) \lambda}^{j \lambda} y(i),\left(1 \leq j \leq \frac{M}{\lambda}\right)
$$

In equation (1): $\lambda$ stands for pulling scale factor, ordinary its value range greater than 1 . If $\lambda$ equal to 1 , then the corresponding function $x_{1}(\lambda)$ stands for the original analytical array, in practical application always select ten above.

(2) Setting mode dimension $n$, and reconfiguring signal sequence. Obtain the vector quantity.

$Y(i)=\{y(i), y(i+1), \ldots, y(i+n-1)\}-y_{0}(i),(i=1,2, \cdots, M-n+1)$

In equation (3): $y_{0}(i)$ is the average value of all elements in reconfiguration order.

$$
y_{0}(i)=\frac{1}{n} \sum_{\gamma=1}^{n-1} u(i+\gamma)
$$

(3) Defining absolute value of maximum D-value between vector quantity $Y(i)$ and $Y(j)$ as $d[Y(i)$, $Y(j)]:$

$$
d[Y(i), Y(j)]=\max _{h=0,1, \cdots, n-1}\left[\left|\left(y(i+h)-y_{0}(i)\right)-\left(y(j+h)-y_{0}(i)\right)\right|\right],(i \neq j)
$$

(4) Introducing fuzzy function $\eta(d[Y(i), Y(j)], s, l)$ to construct the similarity of vector quantity $Y(i)$ and $Y(j)$, the calculated mode

$$
D_{i j}=\eta(d[Y(i), Y(j)], s, l)=e^{-(d[Y(i), Y(j)] / l)^{s}}
$$

In equation (5): $n$ stands for the gradient of exponential function's boundary; $r$ stands for the width of exponential function's boundary.

(5) Basing on similarity to formation the function $\varphi(M, n, r)$,

$$
\varphi(M, n, r)=\frac{1}{M-n} \sum_{i=1}^{M-n}\left(\frac{1}{M-n-1} \sum_{\substack{j=1 \\ j \neq i}}^{M-n} D_{i j}^{n}\right)
$$

(6) Adding the mode dimension to $n+1$ to formation a group of new vector array, and repeating the step of (2), (3) and (4).

$$
\varphi(M, n+1, r)=\frac{1}{M-n} \sum_{i=1}^{M-n}\left(\frac{1}{M-n-1} \sum_{\substack{j=1 \\ j \neq i}}^{M-n} D_{i j}^{n+1}\right)
$$

(7) Using the reconfiguration array $\varphi(M, n, r)$ and $\varphi(M, n+1, r)$ to calculate Fuzzy Entropy.

$$
\operatorname{FE}(M, n, r)=\lim _{M \rightarrow \infty}[\ln \varphi(M, n, r)-\varphi(M, n+1, r)]
$$

In actual signal analysis, subjecting to the length of signal, it always takes advantage of estimating to calculate.

$$
F E(M, n, r)=\ln \varphi(M, n, r)-\ln \varphi(M, n+1, r)
$$

\section{Selection of IMF based on the Index Energy}

In ideal condition, EMD can obtain Intrinsic Mode Function (IMF) which contains single frequency according to different time scale. However, in practical application, due to the end effect and mode mixing, the result of decomposition usually contains overmuch false component. So, in this situation, we not only can obtain signal's inner real feature, but also can increase the calculated amount of feature extraction. In order to solve this problem, we have to effectively distinguish the real component and the false component before the feature extraction.

In the viewpoint of signal analysis, time scale and energy which follows a time scale distribution is very important measurement index. Hence, it provides opportunity to judge signal in different time scale and frequency's energy characteristic. Thereby, it can effectively accomplish the separation of the real component and the false component. For obtaining a group of feature vector quantity that could better reflect signal's energy in different condition, we introduce the concept of 
Index Energy. Combining with the process of EMD, we judges the original signal's energy proportion in the IMF, the specific counting process is as follows:

$$
E(i)=\frac{I_{f_{i}(j)}}{I_{x(j)}}=\sqrt{\frac{\frac{1}{M_{t}} \sum_{j=1}^{M_{t}}\left|f_{i}(j)\right|^{2}}{\frac{1}{N_{t}} \sum_{j=1}^{N_{t}}|x(j)|^{2}}}
$$

In the equation (10): $I_{f i}(j)$ stands for the IMF component's energy coefficient; $I_{x}(j)$ stands for the original vibration signal's energy coefficient; $M_{t}$ stands for IMF component's data length; $N_{t}$ stands for the original vibration signal's data length; $E(i)$ stands for corresponding component's Index Energy. In the process of EMD, the length of IMF component's and original vibration signal is equal. So we can find:

$$
E(i)=\frac{I_{f_{i}(j)}}{I_{x(j)}}=\sqrt{\frac{\sum_{j=1}^{M_{t}}\left|f_{i}(j)\right|^{2}}{\sum_{j=1}^{N_{t}}|x(j)|^{2}}}
$$

Combining with the expression of Index Energy, we can find that the richer signal's feature information IMF contains, the bigger Index Energy is, and the more important of meaning is. What's more, Index Energy is constructed through the inner dynamic relation between the signal in real-time monitoring and subsector. Hence, in some meaning, Index Energy is a process amount [4][5]. It provides condition for judging the information that contained in component dynamically.

\section{Achievement of feature extraction}

Selecting the proposed method to analysis vibration signal in different condition, flow chart of Multi-scale Fuzzy Energy extraction based on Index Energy is as follows.

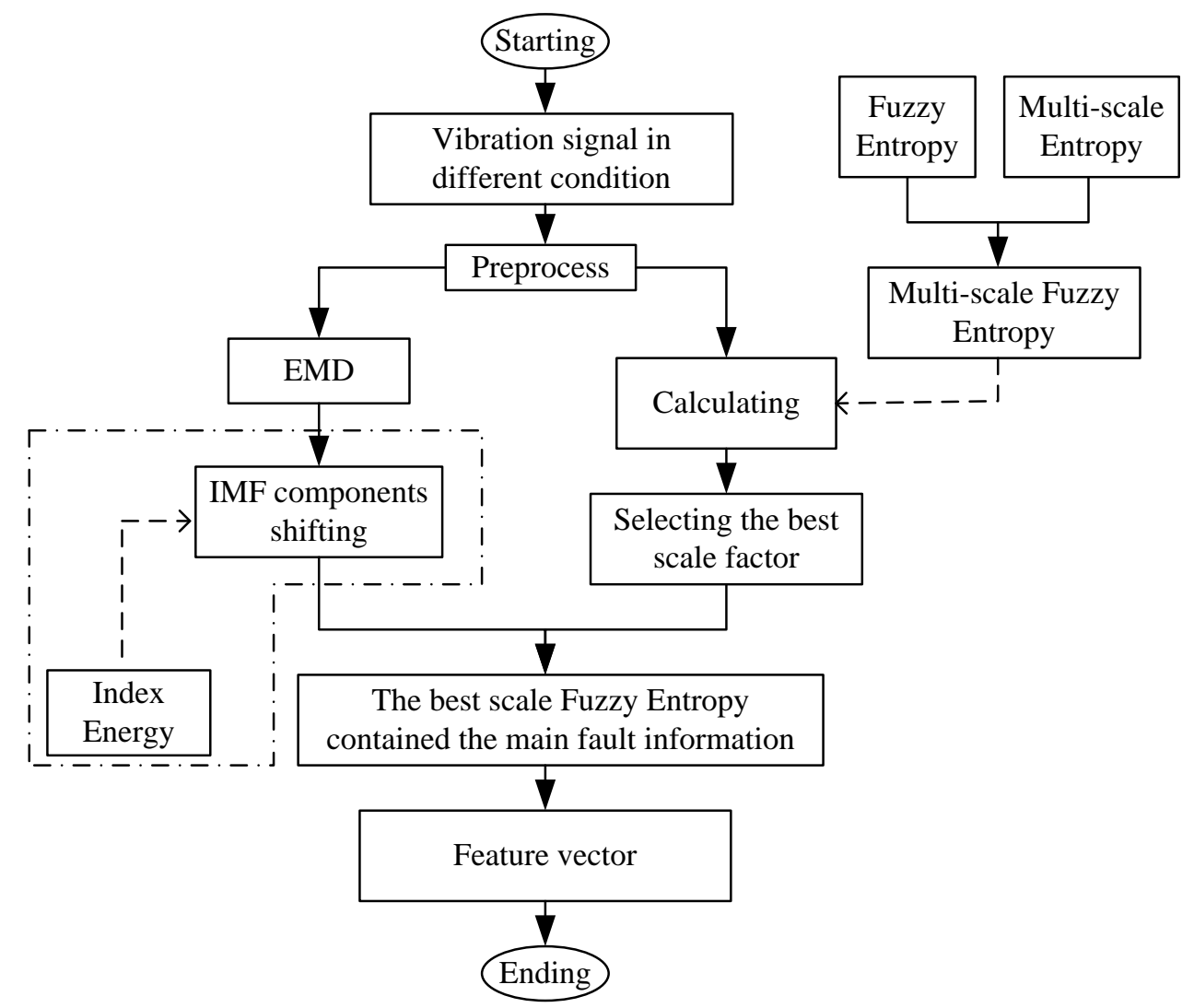

Fig.1 Flow chart of Multi-scale Fuzzy Entropy extraction based on index energy 


\section{Test results}

Disposing the rotor vibration signal with the proposed method, different four condition of fault signal shown in Figure.2. Among them contains rub-impact failure, misalignment failure, looseness failure and normal condition. The analysis result of Multi-scale Fuzzy Entropy is shown in Figure.3.
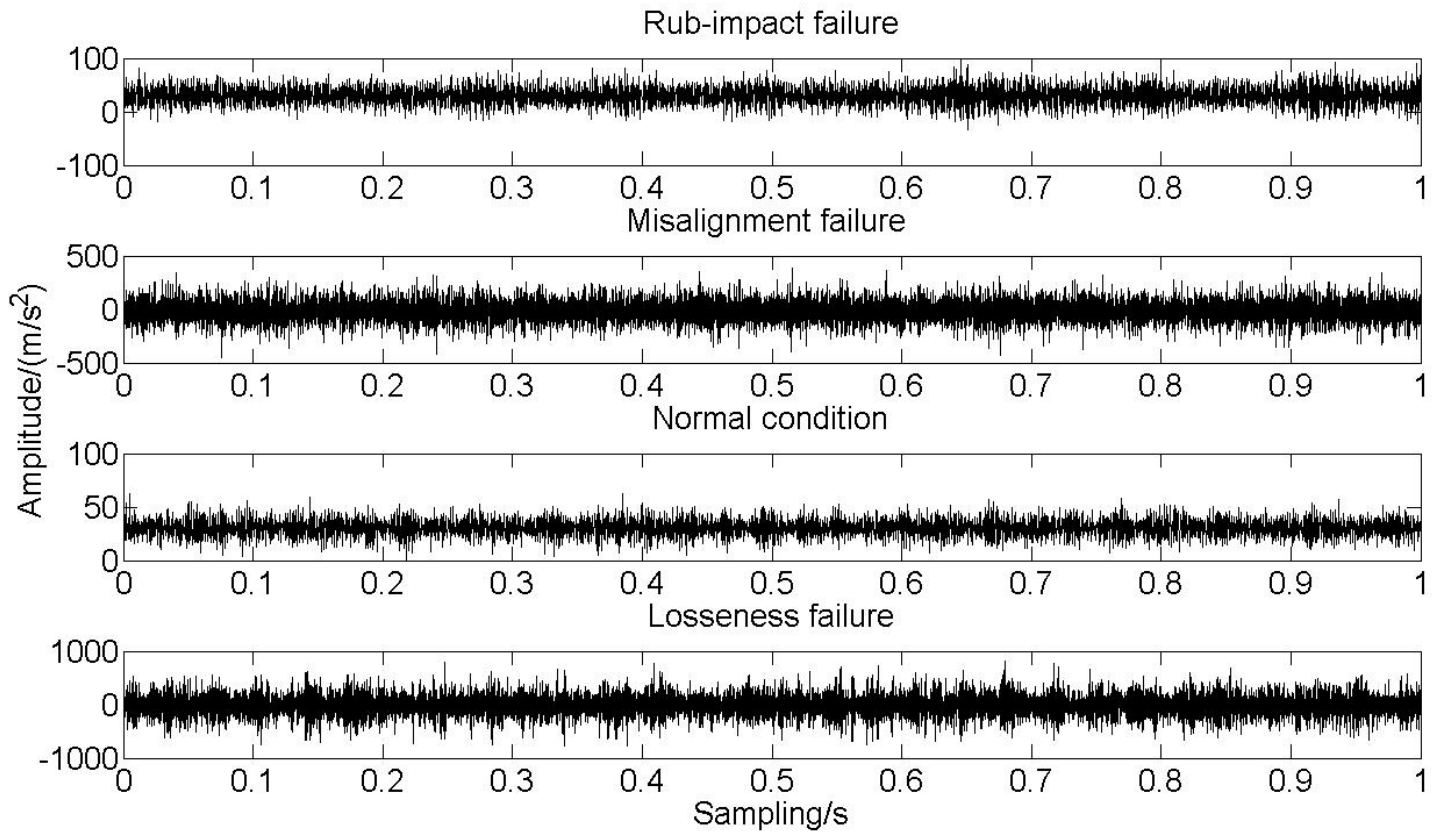

Fig. 2 Time domain waveform under rotor's different four conditions

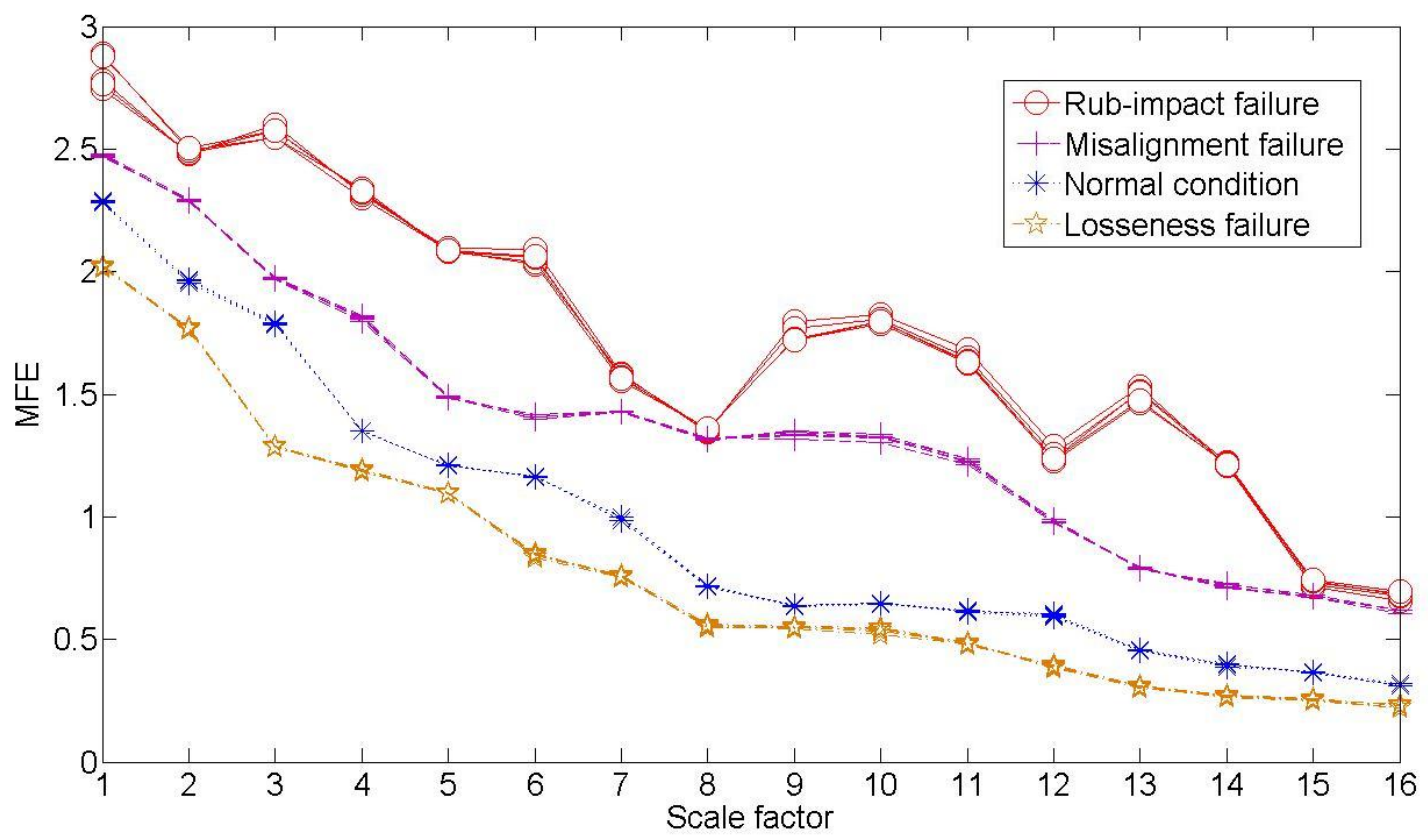

Fig. 3 Multi-scale Fuzzy Entropy (MFE) analysis for rotor’s vibration signals with different condition

Combining with Multi-scale analysis, we can effectively distinguish different condition of vibration signal. For going a step further to analysis the inner feature, we decide to carry out selecting the best scale factor. Calculating difference value of Entropy, thereby shows the difference of different condition. As shown in Tab.1, we can learn the best scale factor is 10 . 
Tab.1 Analysis for distinction degree of samples’ MFE difference

\begin{tabular}{ccccccccccc}
\hline \multirow{2}{*}{ Scale factor } & \multicolumn{3}{c}{ MFE1-MFE2 } & \multicolumn{3}{c}{ MFE2-MFE3 } & \multicolumn{3}{c}{ MFE3-MFE4 } & Sum of \\
\cline { 2 - 10 } mean value \\
\hline 1 & 0.2728 & 0.3128 & 0.2902 & 0.1843 & 0.1874 & 0.1884 & 0.2670 & 0.2603 & 0.2704 & 0.7445 \\
2 & 0.1969 & 0.1933 & 0.2001 & 0.3342 & 0.3362 & 0.3201 & 0.1871 & 0.1837 & 0.1993 & 0.7169 \\
3 & 0.5810 & 0.6194 & 0.6025 & 0.1800 & 0.1936 & 0.1762 & 0.5008 & 0.4972 & 0.5106 & 1.2871 \\
4 & 0.5043 & 0.5099 & 0.5092 & 0.4472 & 0.4568 & 0.4622 & 0.1631 & 0.1643 & 0.1640 & 1.1270 \\
5 & 0.5968 & 0.6051 & 0.5933 & 0.2781 & 0.2817 & 0.2812 & 0.1164 & 0.1186 & 0.1166 & 0.9959 \\
6 & 0.6316 & 0.6854 & 0.6600 & 0.2360 & 0.2431 & 0.2364 & 0.3109 & 0.3313 & 0.3202 & 1.2183 \\
7 & 0.1325 & 0.1567 & 0.1446 & 0.4262 & 0.4283 & 0.4374 & 0.2460 & 0.2466 & 0.2325 & 0.8169 \\
8 & 0.0391 & 0.0277 & 0.0400 & 0.6076 & 0.5956 & 0.5969 & 0.1535 & 0.1685 & 0.1690 & 0.7993 \\
9 & 0.4031 & 0.4459 & 0.4221 & 0.6769 & 0.7120 & 0.7093 & 0.0923 & 0.0968 & 0.0920 & 1.2168 \\
10 & $\mathbf{0 . 4 8 4 5}$ & $\mathbf{0 . 4 9 2 5}$ & $\mathbf{0 . 4 7 9 6}$ & $\mathbf{0 . 6 5 5 8}$ & $\mathbf{0 . 6 8 3 9}$ & $\mathbf{0 . 6 7 5 6}$ & $\mathbf{0 . 1 0 4 3}$ & $\mathbf{0 . 1 2 8 3}$ & $\mathbf{0 . 1 1 3 6}$ & $\mathbf{1 . 2 7 2 7}$ \\
11 & 0.4130 & 0.4497 & 0.4249 & 0.5997 & 0.6225 & 0.6109 & 0.1252 & 0.1297 & 0.1269 & 1.1675 \\
12 & 0.2517 & 0.3094 & 0.2761 & 0.3832 & 0.3811 & 0.3732 & 0.1991 & 0.2098 & 0.2192 & 0.8676 \\
13 & 0.6748 & 0.7390 & 0.7151 & 0.3383 & 0.3430 & 0.3334 & 0.1419 & 0.1453 & 0.1575 & 1.1961 \\
14 & 0.5102 & 0.4963 & 0.5006 & 0.3148 & 0.3142 & 0.3132 & 0.1278 & 0.1334 & 0.1292 & 0.9466 \\
15 & 0.0635 & 0.0435 & 0.0524 & 0.3079 & 0.3064 & 0.3070 & 0.1106 & 0.1146 & 0.1132 & 0.4730 \\
16 & 0.0694 & 0.0351 & 0.0600 & 0.3051 & 0.3088 & 0.3056 & 0.0915 & 0.0923 & 0.0912 & 0.4530 \\
\hline
\end{tabular}

After selecting the best scale factor, we need to combine with the Index energy to search the fault information. Every condition we only list the first 11 IMF. The result is shown in Tab.2. Combining with the best scale factor, we can obtain the discrimination as shown in Tab.3.

Tab.2 The IMF's index energy and its normalization results in different conditions

\begin{tabular}{ccccccccc}
\hline \multirow{2}{*}{ IMF } & \multicolumn{4}{c}{ Index Energy } & \multicolumn{4}{c}{ Normalization Energy } \\
\cline { 2 - 9 } & Rub & Mis & Nor & Los & Rub & Mis & Nor & Los \\
\hline IMF1 & $\underline{\mathbf{0 . 7 6 5 4}}$ & $\underline{\mathbf{0 . 8 5 8 8}}$ & $\underline{\mathbf{0 . 7 2 5 1}}$ & $\underline{\mathbf{0 . 9 4 2 9}}$ & $\underline{\mathbf{0 . 4 3 7 2}}$ & $\underline{\mathbf{0 . 4 8 6 5}}$ & $\underline{\mathbf{0 . 3 9 7 8}}$ & $\underline{\mathbf{0 . 5 9 2 1}}$ \\
IMF2 & $\underline{\mathbf{0 . 6 1 6 4}}$ & $\underline{\mathbf{0 . 4 4 1 7}}$ & $\underline{\mathbf{0 . 6 3 3 5}}$ & $\underline{\mathbf{0 . 2 6 0 4}}$ & $\underline{\mathbf{0 . 3 5 1 4}}$ & $\underline{\mathbf{0 . 2 5 0 2}}$ & $\underline{\mathbf{0 . 3 4 7 5}}$ & $\underline{\mathbf{0 . 1 6 3 5}}$ \\
IMF3 & $\underline{\mathbf{0 . 1 6 1 0}}$ & $\underline{\mathbf{0 . 2 3 8 5}}$ & $\underline{\mathbf{0 . 2 4 6 5}}$ & $\underline{\mathbf{0 . 1 8 6 3}}$ & $\underline{\mathbf{0 . 0 9 1 2}}$ & $\underline{\mathbf{0 . 1 3 5 1}}$ & $\underline{\mathbf{0 . 1 3 5 2}}$ & $\underline{\mathbf{0 . 1 1 7 0}}$ \\
IMF4 & $\underline{\mathbf{0 . 0 6 6 2}}$ & $\underline{\mathbf{0 . 0 8 2 1}}$ & $\underline{\mathbf{0 . 0 9 2 8}}$ & $\underline{\mathbf{0 . 0 7 0 0}}$ & $\underline{\mathbf{0 . 0 3 8 0}}$ & $\underline{\mathbf{0 . 0 4 6 5}}$ & $\underline{\mathbf{0 . 0 5 0 9}}$ & $\underline{\mathbf{0 . 0 4 4 0}}$ \\
IMF5 & 0.0407 & 0.0398 & 0.0452 & 0.0435 & 0.0017 & 0.0226 & 0.0248 & 0.0273 \\
IMF6 & 0.0300 & 0.0302 & 0.0278 & 0.0263 & 0.0169 & 0.0171 & 0.0152 & 0.0165 \\
IMF7 & 0.0270 & 0.0225 & 0.0150 & 0.0232 & 0.0142 & 0.0128 & 0.0082 & 0.0146 \\
IMF8 & 0.0159 & 0.0169 & 0.0131 & 0.0152 & 0.0091 & 0.0096 & 0.0072 & 0.0095 \\
IMF9 & 0.0122 & 0.0123 & 0.0083 & 0.0138 & 0.0063 & 0.0070 & 0.0046 & 0.0087 \\
IMF10 & 0.0083 & 0.0166 & 0.0051 & 0.0073 & 0.0053 & 0.0094 & 0.0028 & 0.0046 \\
IMF11 & 0.0109 & 0.0059 & 0.0105 & 0.0036 & 0.0067 & 0.0033 & 0.0057 & 0.0023 \\
\hline
\end{tabular}

Tab. 3 The first four IMFs’ Optimum Fuzzy Entropy(OFE) in different conditions

\begin{tabular}{|c|c|c|c|c|}
\hline \multirow[t]{2}{*}{ Sample } & \multicolumn{4}{|c|}{$\begin{array}{l}\text { The best scale of MFE } \\
\text { (IMF1,IMF2, } \\
\text { IMF3,IMF4) }\end{array}$} \\
\hline & Rub & Mis & Nor & Los \\
\hline 1 & $\begin{array}{l}(3.3675,1.3856, \\
1.1935,0.6772)\end{array}$ & $\begin{array}{c}(5.2623,2.0159, \\
0.8048,0.5665)\end{array}$ & $\begin{array}{c}(1.4578,0.9547, \\
0.6840,0.5007)\end{array}$ & $\begin{array}{l}(1.3454,0.6851, \\
0.5553,0.4287)\end{array}$ \\
\hline 2 & $\begin{array}{c}(3.3691,1.3895, \\
1.1930,0.6737)\end{array}$ & $\begin{array}{c}(5.2585,2.0489 \\
0.8089,0.5640)\end{array}$ & $\begin{array}{c}(1.4522,0.9563, \\
0.6821,0.5113)\end{array}$ & $\begin{array}{c}(1.3265,0.6837 \\
0.5625,0.4237)\end{array}$ \\
\hline 3 & $\begin{array}{c}(3.3752,1.3895 \\
1.1927,0.6827)\end{array}$ & $\begin{array}{c}(5.2562,1.9881 \\
0.8111,0.5653)\end{array}$ & $\begin{array}{c}(1.4607,0.9646, \\
0.6901,0.5068)\end{array}$ & $\begin{array}{c}(1.3117,0.6823, \\
0.5597,0.4172)\end{array}$ \\
\hline 4 & $\begin{array}{c}(3.3744,1.3879, \\
1.1920,0.6753)\end{array}$ & $\begin{array}{c}(5.2617,1.9910 \\
0.8196,0.5598)\end{array}$ & $\begin{array}{c}(1.4614,0.9519 \\
0.6935,0.5165)\end{array}$ & $\begin{array}{l}(1.3277,0.6854 \\
0.5629,0.4231)\end{array}$ \\
\hline 5 & $\begin{array}{c}(3.3668,1.3907, \\
1.1904,0.6742)\end{array}$ & $\begin{array}{c}(5.2589,2.0258, \\
0.8219,0.5617)\end{array}$ & $\begin{array}{c}(1.4540,0.9573, \\
0.6857,0.5044)\end{array}$ & $\begin{array}{c}(1.3238,0.6894 \\
0.5417,0.4350)\end{array}$ \\
\hline
\end{tabular}




\begin{tabular}{cccccc}
\hline \multirow{2}{*}{6} & $(3.3775,1.3835$, & $(5.2638,2.0811$, & $(1.4688,0.9554$, & $(1.3197,0.6946$, \\
& $1.1859,0.6727)$ & $0.8161,0.5702)$ & $0.6927,0.5109)$ & $0.5457,0.4393)$ \\
7 & $(3.3513,1.3912$, & $(5.2704,1.9847$, & $(1.4543,0.9587$, & $(1.3224,0.6945$, \\
& $1.1905,0.6741)$ & $0.8219,0.5715)$ & $0.6888,0.5134)$ & $0.5448,0.4326)$ \\
8 & $(3.3577,1.3839$, & $(5.2683,2.0216$, & $(1.4627,0.9601$, & $(1.3431,0.6828$, \\
& $1.1882,0.6626)$ & $0.8275,0.5694)$ & $0.6894,0.5064)$ & $0.5641,0.4328)$ \\
9 & $(3.3701,1.3861$, & $(5.2746,2.0581$, & $(1.4582,0.9634$, & $(1.3536,0.7023$, \\
& $1.1850,0.6889)$ & $0.8195,0.5583)$ & $0.6926,0.5126)$ & $0.5629,0.4409)$ \\
10 & $(3.3629,1.3901$, & $(5.2569,2.0466$, & $(1.4704,0.9473$, & $(1.3486,0.6937$, \\
& $1.1859,0.6761)$ & $0.8327,0.5614)$ & $0.6738,0.5131)$ & $0.5714,0.4368)$ \\
\hline
\end{tabular}

\section{Conclusion}

Combining with the theory of Entropy, we gave a deeply research on the method of feature extraction, and put forward a method of Multi-scale Fuzzy Entropy based on Index Energy. Utilizing it to deal with the rotor's vibration signal, the result proves that the feature vector can effectively reflect the change of rotor's condition. And the distinction degree is very obvious to catch the change of switching transients.

\section{References}

[1] Pincus S M. Approximate entropy as a measure of system complexity[J]. Proceedings of National Academy of Sciences, 1991, 88(6): 2297-2301.

[2] Richman J S, Moorman J R. Physiological time-series analysis using approximate entropy and sample entropy[J]. American Journal of Physiology-Heart and Circulatory Physiology, 2000, 278: 2039-2049.

[3] Chen Weiting, Wang Zhizhong, XieHongbo, et al. Characterization of surface EMG signal based on fuzzy entropy[J]. IEEE Transactions on Neural Systems and Rehabilitation Engineering, 2007, 15(2): 267-272.

[4] Costa M, Goldberger A L, Peng C K. Multiscale entropy analysis of biological signals[J]. Physical Review E, 2005, 71: 1-18.

[5] Zheng Jinde, Chen Minjun, Cheng Junsheng etc. Multiscale fuzzy entropy and its application in rolling bearing fault diagnosis[J]. Journal of Vibration Engineering, 2014,27(1):145-151.

[6] Li Linfeng, Zhao Jun, Guo Tiantai, Song Yuqian. Gear Fault Diagnosis based on EEMD, Fuzzy Entropy and SVM[J]. Journal of Mechanical Transmission, 28(2):147-151. 